\title{
A LARGO PLAZO: PROYECTOS NARRATIVOS TRANSTEMPORALES EN LA ARGENTINA CONTEMPORÁNEA
}

\author{
Sandra Contreras \\ Instituto de Estudios Críticos en Humanidades, IECH \\ Universidad Nacional de Rosario \\ Consejo Nacional de Investigaciones Científicas y Técnicas, CONICET \\ Argentina \\ sandracontreras123@gmail.com \\ Orcid: 0000-0003-469I-866I
}

\section{Alejandra Laera}

Universidad de Buenos Aires

Consejo Nacional de Investigaciones Científicas y Técnicas, CONICET

Argentina

alelaera@gmail.com

Orcid: 0000-0002-4317-9683

Fecha de recepción: 30/09/202I | Fecha de aceptación: 15/I0/202 |

\begin{abstract}
Resumen: Mientras las experiencias vigentes en torno al tiempo parecen dominadas por la velocidad, la obsolescencia inmediata, el cortoplacismo, los trabajos que componen este dossier reúnen materiales estéticos heterogéneos en una serie cultural que esboza modos alternativos de procesar, habitar, reimaginar la temporalidad.A través del análisis propuesto, obras literarias, cinematográficas y teatrales de la Argentina contemporánea entran en diálogo en función de una apuesta común por el largo plazo, por la larga duración como unidad de medida para sus respectivos proyectos narrativos. Así, el tiempo recupera la centralidad extraviada en las imaginaciones narrativas -y las reflexiones críticas-, pero lo hace bajo las formas del cuestionamiento de su percepción, la discontinuidad de sus ritmos y cronologías, la interrogación sobre lo que significa habitar el tiempo y la apertura a las distintas temporalidades que se acoplan, descomponen o soslayan detrás de ese uso del singular.
\end{abstract}

Palabras claves: Larga duración; temporalidades; literatura argentina contemporánea; cine argentino contemporáneo; teatro argentino contemporáneo. 


\title{
Long-term: Transtemporal Narrative Projects \\ in Contemporary Argentina
}

\begin{abstract}
While current experiences around time seem dominated by speed, immediate obsolescence and the short-termism, the works that make up this dossier bring together heterogeneous aesthetic materials in a cultural series that outlines alternative ways of processing, inhabiting, reimagining temporality. Through the proposed analysis, literary, cinematographic and theatrical works of contemporary Argentina enter into a dialogue based on a common commitment to the long term, to long duration as a unit of measurement for their narrative projects. Thus, time recovers the lost centrality in narrative imaginations -and critical reflections-, but it does so in the form of questioning its perception, the discontinuity of its rhythms and chronologies, the interrogation of what it means to inhabit time and the opening to the different temporalities that are coupled, descompose or ignored behind this use of the singular.
\end{abstract}

Keywords: Long duration; Temporalities; Contemporary Argentine Literature; Contemporary Argentine Cinema; Contemporary Argentine Theather.

\section{Longo prazo: projetos narrativos transtemporais na Argentina contemporânea}

Resumo: Enquanto as experiências atuais em torno do tempo parecem dominadas pela velocidade, obsolescência imediata, curto prazo, os artigos que compõem este dossiê reúnem materiais estéticos heterogêneos em uma série cultural que delineia formas alternativas de processar, habitar, reimaginar a temporalidade.Através da análise proposta, as obras literárias, cinematográficas e teatrais da Argentina contemporânea dialogam a partir de um compromisso comum com o longo prazo, com a longa duração como unidade de medida de seus respectivos projetos narrativos. Assim, o tempo recupera a centralidade perdida na imaginação narrativa -e na reflexão crítica-, mas o faz na forma de questionar sua percepção, a descontinuidade de seus ritmos e cronologias, a interrogação sobre o que significa habitar o tempo e a abertura às diferentes temporalidades que se acopla, se decompõem ou se ignora por trás desse uso do singular.

Palavras chaves: Longa duração;Temporalidades; literatura argentina contemporânea; cinema argentino contemporâneo; teatro argentino contemporâneo

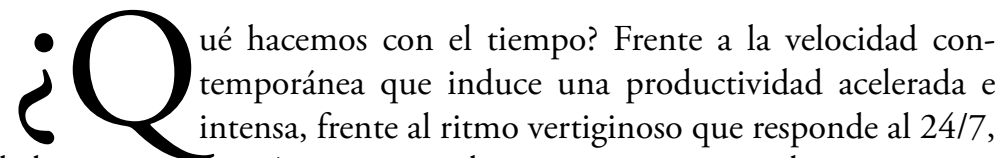

frente a la hiperconcentración por intervalos que exige un recambio motivacional constante, el tiempo se llena de acciones que parecen llenarlo a la vez que alargarlo, ocuparlo a la vez que rebalsarlo. Sin embargo, frente al cortoplacismo, la obsolescencia, el aburrimiento, persiste, en un conjunto de narraciones de escritorxs y artistas visuales, el desafío del largo plazo, el interés continuado en un mismo proyecto, una concentración sostenida y a la vez vital. Se trata de obras literarias, cinematográficas y teatrales que apuestan a la larga duración.

Pero entonces: ¿cómo imaginamos ese tiempo? Si un proyecto narrativo de largo plazo pone en cuestión, con su propia ambición, la relación discontinua entre presente y futuro mayormente asociada a la inflexión capitalista contemporánea, lo hace también porque entra en tensión con la noción misma de tiem- 
po. En vez de un tiempo lineal, progresivo, universal, estos proyectos juegan narrativamente con la duración: cuestionan, de un modo diverso a como lo hizo la novela del siglo XX, la cronología, el tiempo histórico, el tiempo único. Se trata de una imaginación en la que diversos tiempos pueden convivir o superponerse o confundirse, pueden discontinuarse o descomponerse. En estos proyectos narrativos a largo plazo el tiempo se abre al juego de las temporalidades y la imaginación se vuelve transtemporal.

\section{Proyectos a largo plazo}

Como se sabe, "larga duración" es el término acuñado por Fernand Braudel para dar cuenta de aquellas permanencias-ciertos marcos geográficos, realidades biológicas, encuadramientos mentales- que el tiempo tarda enormemente en desgastar y en transformar: estructuras estables o perdurables que se verifican en el tiempo profundo de la geología, pero también en el del inmenso campo de la realidad social y cultural. Si en los años 50 el concepto apuntó a fundamentar un método de observación, común a las ciencias sociales, contra la historia acontecimental, la larga duración por la que apuestan Jo Guldi y David Armitage en The History Manifesto es proclamada en 2014 como un "llamado a las armas" contra el "espectro" del cortoplacismo que amenaza a nuestro tiempo, una urgencia por reconsiderar lo contemporáneo en función de la búsqueda de sentidos y de la interpretación que solo la experiencia histórica hace posible. Nuestra perspectiva, sin embargo, no es historiográfica; no se trata de proyectar en las obras una apuesta por recuperar el espesor de la historia frente al presentismo monocromo en que se desintegran los sentidos. Lo que nos interesa en ciertos proyectos artísticos a largo plazo son sus formas singulares de practicar e imaginar la duración, de habitar el tiempo y el presente mismo.

Desde luego, todas las variantes de la narrativa de larga duración -gran novela, summa, saga o comedia humana, novela total o monumento novelesco-se identifican ampliamente en la gran tradición moderna, también en la vanguardista, y constituyen inclusive uno de sus signos distintivos. Lo que hoy llama nuestra atención en ciertas obras, a punto tal que se vuelve un tópico ineludible comenzar a hablar de ella refiriéndonos a su extensión -por ejemplo, las catorce horas de La flor (2018) de Mariano Llinás, o las tres mil quinientas páginas de Mi lucha (2009-2011) de Karl Ove Knausgaard- es una duración que aparece, se presenta o se percibe como desmesurada en el contexto de una cultura cuya relación misma con el tiempo se ha modificado de modo sustancial. Esa relación, signada por la velocidad de una aceleración que desde la segunda mitad del siglo XX tendió a suprimirlo junto con sus experiencias (Virilio) es una experiencia cultural donde la expansión de la sociedad de consumo, el rápido avance de la tecnología y las redes de conexión interpersonal hicieron del presente un instante listo para ser reemplazado por el siguiente, y donde lo nuevo es, a diferencia del tiempo cronológico de las naciones, las finanzas y la industria, "el abandono 
radical de la pretensión de que el tiempo se acople a cualquier proyecto a largo plazo, incluso a las fantasías de 'progreso' o desarrollo" (Crary). Si además, lo que define el régimen del 24/7 es la inscripción de la vida en una duración sin interrupción a partir de un tiempo indiferenciado que socava las distinciones noche-día, luz-oscuridad, trabajo-ocio (Crary), si esa continuidad indiscriminada (el trabajar sin descanso) se vincula con el insomnio como síntoma complejo de la sociedad contemporánea, las narrativas de larga duración hoy deberían pensarse también como disrupciones en el marco de una era en que las formas de atención, y de lectura, se ven sensiblemente afectadas. Como si, al trabajar en la complejidad de proyectos desmesurados, ciertas narrativas de larga duración hicieran hoy particularmente visible, al modo de un subrayado, no tanto el tamańo implícito en la idea de "gran obra" como el volumen de tiempo invertido en su factura, la cantidad de tiempo que demanda su consumo.

Una forma de percibir esos modos de la extensión es la de la modernidad crítica. Por ejemplo, la de Beatriz Sarlo (2004) cuando entiende que asistir a la ejecución del cuarteto de cuerdas $\mathrm{n}^{\circ} 2$ de Morton Feldman durante cinco horas o a una lectura del texto completo de Moby Dick durante veinticuatro en el teatro constituyen experiencias en las que la duración desacostumbrada exige unas fuerzas extraordinarias para acompañar, hasta el final, el cumplimiento de la pieza: una prueba de resistencia corporal y un desafío de atención que tanto ejecutantes como asistentes sortean no por un simple deseo de escucha, según el "plegadizo orden del gusto", sino como una decisión de seguir la señal de "algo del orden estético duro" que, dice, es el signo incontestable de que el tiempo puede todavía vivirse como sustancia estética en el mundo contemporáneo. En otro sentido, artistas de la desmesura como Rafael Spregelburd (2008) pueden vincularse con la extensión de un modo menos atento a las pruebas del arte que a interpretar, en cambio, la particular economía en que se constituye la larga duración: imaginar, por ejemplo, las diez semanas en que se sucedieron los diez episodios de Bizarra (2002) o las tres horas y media que requiere la puesta en escena de La estupidez (2003) como emergentes de un "capitalismo flagrante y alienante" que en la Argentina de los tempranos años 2000 llevó a "la gente" a "una natural desconfianza ante cualquier medida institucionalizada" y a producir, tanto como a acompañar, en una relación "loca" con el mercado, obras de duración desaforada. La larga duración, entonces, practicada e imaginada por un dramaturgo argentino como una economía ficcional de la extensión, disruptiva en la era del nuevo capitalismo, solo que desde una perspectiva que además tiene la ventaja de situar la emergencia de esa economía en el marco de un neoliberalismo que aquí, en América Latina o en el tiempo otro de "Buenos Aires año 2000", para decirlo con Josefina Ludmer (2010), se acelera hasta hacer estallar la temporalidad estatal.

Por otra parte, entendidas como una economía ficcional, en el sentido ahora de una distribución de quantos de energía, ejercicios de escritura como el que Mario Levrero llama para sí "novela luminosa" y que acomete, entre interrupcio- 
nes, recomienzos y continuaciones, a lo largo de dieciocho años -entre "Diario de un canalla" (1992), El discurso vacio (1997) y La novela luminosa (2005)-, o como los de Cuadernos de lengua y literatura (2000-2021) de Mario Ortiz en que el pasaje de la poesía a la prosa es indisociable de una experimentación con los huecos del tiempo, pueden ser leídos como proyectos que, en su devenir y más allá del género y de su deconstrucción, adoptan la novela o el relato como práctica: como marco, como pulsión, como vía de exploración para (volver a) poner en marcha la escritura después de la experiencia de la parálisis o del agotamiento de la imaginación. Fuera ahora de la pregunta por la escala, y en un sentido por completo diferente al señalado por Amitav Ghosh, cuando al pensar la relación entre tiempo geológico profundo e imaginación literaria dice que "la larga duración no es el territorio de la novela" (103), ejercicios como estos son señales de la forma en que ciertas narrativas contemporáneas de larga duración también pueden ser pensadas, en tanto inflexión contemporánea del entusiasmo, como una nueva forma de investir el acto de novelar después de la gran novela moderna.

\section{Imaginación narrativa transtemporal}

El largo plazo de ciertos proyectos narrativos contemporáneos involucra al tiempo no solo en lo que respecta a su duración, desafiando cortoplacismos y obsolescencias, sino que es la propia noción de tiempo la que se ve afectada al ser (re)puesta en el centro de la imaginación narrativa como no lo era desde la época de entronización de la gran novela moderna que precisaba de la sucesión temporal o bien para la organización secuencial de sus historias realistas o bien para la reconstrucción de los desórdenes enunciativos de los relatos experimentales. La crisis de la idea de mundo en los últimos años e incluso la acechanza creciente de final de mundo, con el develamiento fantasmático de un mundo estable cuya estructura simbólica moderna era afectiva y universal debido a conexiones globales, traducciones, interacciones y desplazamientos (Siskind), ha estado acompañada de la crisis de la imaginación global que configuró predominantemente la narrativa de las décadas recientes. Junto con la experiencia literaria de esa crisis de mundo, ha surgido una imaginación literaria posglobal que encuentra su impulso en el tiempo, que encuentra en el tiempo una materia con la cual desafiar la racionalidad de raíz moderna para pensar el mundo, la historia y también la vida.

Por un lado, lo hace al proponerlo como material privilegiado de sus elaboraciones y resoluciones narrativas, tal como ocurre en varias trilogías del fin del mundo como las de Oliverio Coelho y Rafael Pinedo, en novelas muy diferentes como El absoluto (2016) de Daniel Guebel y La familia (2014) de Gustavo Ferreyra que vuelven a la saga, o en el proyecto de larga duración sobre la Guerra de Malvinas que Lola Arias planteó en diversos formatos alrededor de Campo minado (2016-2018): el tiempo es el motivo sobre el que gira la trama, el que atraviesa las historias y la Historia, el que sutura o astilla las vidas, es un motivo 
intrínseco a los modos que asume el relato a la vez que un motivo de la narración. Por otro lado, es una imaginación literaria posglobal que desglosa la noción de tiempo y en vez de apostar ya modernamente a un tiempo único, concibe la distinción de temporalidades en convivencia o eclosión (tal como se adelantó con anclaje puramente ficcional en Los Sorias (1998) de Alberto Laiseca o como culminó con un anclaje documental ficcionalizado en Los diarios de Emilio Renzi (2015-2017) de Ricardo Piglia o cómo transcurre en la "novela de la poesía" de Tamara Kamenszain): el tiempo que pasa no está atado inherentemente al calendario (un calendario que habilita mundos paralelos e incluso cronologías paralelas pero nunca otros tiempos) sino que ofrece múltiples interpretaciones de ese pasaje (Latour). Así, se trata de una imaginación literaria transtemporal que juega con las temporalidades al proponer tiempos que se discontinúan, se descomponen, se repiten, tiempos que se aceleran y desaceleran, tiempos que conviven, se superponen, se confunden. Por medio de esos juegos, o a través de ellos, estas narrativas transtemporales de largo plazo proponen una salida a los problemas planteados en y por el espacio, el mundo, la vida. En estos proyectos narrativos a largo plazo el tiempo, desafiando heterocrónicamente la mera progresión y la historicidad moderna, se abre al juego de las temporalidades.

Si la ciencia acepta que el tiempo no es único, que hay duraciones diferentes para cada trayectoria, si hay ritmos diferentes del transcurrir según el lugar y la velocidad, si ha probado que no hay una orientación dada entre pasado y futuro (Rovelli), ¿qué aporta la literatura a esa comprensión del mundo como conjunto de eventos y procesos que nos permite captarlo en la contemporaneidad? Ahí mismo es donde se ubican, para nosotras, los proyectos narrativos transtemporales de largo plazo. Ahí mismo donde la imaginación literaria se abre a la exploración especulativa, a la práctica, a la escritura y la performance visual, a la puesta en escena, y se reinventa y se repolitiza para comprender algo del presente.

\section{Descripción del dossier}

La propuesta de este dossier surge de un trabajo de equipo. En los últimos dos años dirigimos un trabajo de investigación sobre proyectos narrativos transtemporales de larga duración en la Argentina contemporánea que incluía materiales escritos, audiovisuales y performáticos muy diversos ${ }^{1}$. A través de las reuniones grupales y de nuestro propio intercambio teórico y crítico fue tomando forma la iniciativa de pensar y leer un conjunto particular de objetos culturales que mostraran el alcance de nuestra hipótesis acerca de un modo particular de concebir el tiempo y elaborar temporalidades en la narrativa contemporánea.

1 El proyecto, radicado en el Instituto de Estudios Críticos en Humanidades (IECH, CONICETUNR), se titula "Proyectos transtemporales: narrativas de larga duración en la literatura, el cine y el teatro argentinos contemporáneos" y es financiado por la Agencia Nacional de Promoción Científica y Tecnológica como PICT 2017-2339. 
Desde las propuestas iniciales a los artículos que hoy presentamos hubo conversaciones, cambios, agregados, reescrituras. Hubo procesos textuales, por lo tanto, pero también eventos históricos que nos hicieron volver a pensar la idea de tiempo, la noción de larga duración y la imaginación narrativa una y otra vez. De esa experiencia compleja surge el resultado de este dossier que agrupamos en tres zonas. "Tiempos de vida" focaliza en las capas temporales de dos obras muy diferentes, los tres volúmenes de Los diarios de Emilio Renzi de Ricardo Piglia y los últimos libros de Tamara Kamenszain, ambas producidas en el transcurrir de una vida (Alejandra Laera, "Tiempo de la vida, tiempos de la ficción: Los diarios de Emilio Renzi de Ricardo Piglia"; Sandra Contreras, "Tempo, escala, tiempos: el relato de larga duración de Tamara Kamenszain"). Por su parte, "Tiempos visuales" plantea la cuestión de la larga duración en dos propuestas cinematográficas casi divergentes como la de Mariano Llinás y la de José Celestino Campusano, en un proyecto biodramático de formatos variados como el de Lola Arias sobre Malvinas y en la serie de "los finales" del teatro de Rafael Spregelburd (Nicolás Suárez y Marcos Zangrandi, "Cómo filmar juntos. Transtemporalidades y variaciones de lo extenso en el cine de Llinás y Campusano"; Lara Segade, "Una imagen que no se olvida: trauma y anacronismo en el proyecto biodramático de Lola Arias sobre la guerra de Malvinas"; María Fernanda Pinta, El fin de Europa (y de lo que no es Europa). Motivos y versiones del final según Rafael Spregelburd'). Finalmente, la tercera parte, "Temporalidades de la ficción”, reúne dos lecturas focalizadas en la potencia transtemporal de la imaginación literaria en la novela de culto de Laiseca y en varias trilogías recientes (Cristian Molina, "Una máquina del tiempo lentísima. Los sorias de Alberto Laiseca"; Mariana Catalin, "Los tiempos del final"). Las tres partes, con los artículos que las componen, creemos, incursionan en algunos de los interrogantes que hoy nos plantea la experiencia del tiempo y, sobre todo, proponen pensar en los desafíos narrativos con los que la literatura, el teatro, y el cine buscan interpelarlo en el presente.

\section{Referencias bibliográficas}

Braudel, Fernand. "La larga duración". La historia y las ciencias sociales. Madrid, Alianza, 1968 [1958].

Crary, Jonathan. 24/7. El capitalismo tardío y el fin del sueño. Buenos Aires, Paidós, 2015 [2013].

Ghosh, Amitav. The Great Derangement. Climate Change and the Unthinkable. London, Penguin Books, 2016.

Guldi Jo y David Armitage. The History Manifesto, Cambridge: Cambridge University Press, 2014. 
Latour, Bruno. Nunca fuimos modernos. Ensayos de antropología simétrica. Buenos Aires, Siglo XXI Editores, 2012 [1991].

Rovelli, Carlo. El orden del tiempo. Barcelona, Anagrama, 2018 [2017].

Siskind, Mariano. "Towards a cosmopolitanism of loss: an essay about the end of the world". World Literature, Cosmopolitanism, Globality. Beyond, Against, Post, Otherwise. Gesine Müller y Mariano Siskind editores. Berlin, De Gruyter, 2019, 205-235.

Sarlo, Beatriz. "La extensión”. Punto de Vista, Año XXVII, No 78, abr. 2004.

Spregelburd, Rafael. "Todo es relativo. Pero esto depende. Reflexiones sobre los caminos del teatro a la complejidad, el extrańamiento y un orden abierto al caos". Otra parte, № 15, primavera 2008.

Virilio, Paul. Velocidad y política, Buenos Aires, La Marca, 2006. 